\title{
La Bibliothèque nationale de Russie à la veille de son bicentenaire
}

\section{The National Library of Russia on the Eve of its Bicentennial La Biblioteca Nacional de Rusia en vísperas de su bicentenario}

\section{Vladimir N. Zaïtsev}

Volume 39, numéro 2, avril-juin 1993

Les bibliothèques nationales

URI : https://id.erudit.org/iderudit/1028738ar

DOI : https://doi.org/10.7202/1028738ar

Aller au sommaire du numéro

Éditeur(s)

Association pour l'avancement des sciences et des techniques de la documentation (ASTED)

ISSN

0315-2340 (imprimé)

2291-8949 (numérique)

Découvrir la revue

Citer cet article

Zaïtsev, V. N. (1993). La Bibliothèque nationale de Russie à la veille de son bicentenaire. Documentation et bibliothèques, 39(2), 69-74.

https://doi.org/10.7202/1028738ar
Résumé de l'article

La Bibliothèque nationale de Russie, une des plus grandes bibliothèques du monde, est issue de la Bibliothèque publique impériale, fondée à Saint-Pétersbourg en 1795. À la veille de son bicentenaire, la Bibliothèque affronte de grandes difficultés, surtout pour ce qui est du développement des collections, mais elle inaugure avec confiance une nouvelle phase de son développement : construction d'un nouvel édifice, développements informatiques et révisions législatives.
Tous droits réservés (c) Association pour l'avancement des sciences et des techniques de la documentation (ASTED), 1993
Ce document est protégé par la loi sur le droit d'auteur. L'utilisation des services d'Érudit (y compris la reproduction) est assujettie à sa politique d'utilisation que vous pouvez consulter en ligne. 


\title{
La Bibliothèque nationale de Russie à la veille de son bicentenaire
}

\author{
Vladimir N. Zaitsev \\ Directeur général \\ Bibliothèque nationale de Russie
}

La Bibliothèque nationale de Russie, une des plus grandes bibliothèques du monde, est issue de la Bibliothèque publique impériale, fondée à Saint-Pétersbourg en 1795. À la veille de son bicentenaire, la Bibliothèque affronte de grandes difficultés, surtout pour ce qui est du développement des collections, mais elle inaugure avec confiance une nouvelle phase de son développement: construction d'un nouvel édifice, développements informatiques et révisions législatives.

\section{The National Library of Russia on the Eve of its Bicentennial}

The National Library of Russia, one of the largest in the world, was born of the Imperial Public Library, founded in St. Petersburg in 1795. On the eve of its bicentennial, the National Library faces grave difficulties especially in the area of collections development. The authorities have confidence in the next phase of its development: the construction of new building, computer technology and revisions to the existing legis/ation.

\section{La Biblioteca Nacional de Rusia en visperas de su bicentenario}

La Biblioteca Nacional de Rusia, una de las más grandes del mundo, proviene de la Biblioteca Pública Imperial, fundada en San Petersburgo en 1795. En vísperas de su bicentenario, la Biblioteca se enfrenta a grandes dificultades, sobre todo en cuanto al desarrollo de las colecciones. A pesar de todo, inaugura con confianza una nueva etapa de su desarrollo: la construcción de un nuevo edificio, desarrollos informáticos, revisiones legislativas.
La Russie compte plus de 150000 bibliothèques offrant leurs services à toutes les couches de la population. La Bibliothèque d'État de Russie (BER) à Moscou et la Bibliothèque nationale de Russie (BNR) à Saint-Pétersbourg sont les plus grandes.

La Bibliothèque nationale de Russie, doyenne des bibliothèques publiques de l'État, célébrera son bicentenaire en 1995. Par l'importance et le volume de ses collections et par son service à la clientèle, elle s'avère une des plus grandes bibliothèques au monde. Fondée en 1795 à Saint-Pétersbourg par Catherine II, qui fut une grande réformatrice de la Russie, elle a été appelée Bibliothèque publique impériale (BPI). «Rassembler dans la Bibliotheque publique tous les livres russes...", tel était le but de l'Impératrice qui a aussi défini le futur statut de bibliothèque nationale en formulant son objectif principal: «devenir le dépót de tout ce qui représente la contribution faite par les Russes à la trésorerie humaine de la pensée, de la science et des belles-lettres».

En 1810, Alexandre 1er, petit-fils de Catherine II, a promulgué l'acte qui mettait la Bibliothèque publique impériale à la disposition de tous les sujets russes et qui instaurait le dépôt légal, permettant de réclamer deux exemplaires gratuits de toutes les publications issues de n'importe quelle imprimerie dans les limites de l'Empire. C'est ainsi que dès sa fondation, le statut de la Bibliothèque publique a comporté les caractéristiques principales qui conservent toujours leur importance: rayonnement à l'échelle nationale, responsabilité de la conservation du patrimoine documentaire national et dispensation de services à tous les citoyens.

La Bibliothèque publique impériale a exercé une influence énorme sur le développement de la vie intellectuelle en Russie. Elle est devenue un pôle important pour la culture, la science et l'instruction russes, et les noms des plus éminents écrivains, savants, militants de la cause publique sont étroitement liés à son activité. L'apport de l'institution au domaine de la bibliothéconomie est également remarquable.

Les fonds de la Bibliothèque nationale de Russie totalisent 31 millions d'unités physiques dont plus de 5 millions en langues étrangères. En accomplissant sa mission majeure qui consiste à collectionner, conserver et communiquer au lecteur ce qui constitue le patrimoine national des imprimés, la Bibliothèque nationale de Russie a constitué la plus grande collection de documents en langue russe. II faut aussi mentionner les efforts déployés afin de rassembler, de conserver et d'étudier les manuscrits nationaux, qu'il s'agisse des chroniques, des chartes de l'ancienne Russie, des manuscrits ou des autographes de personnalités éminentes de la culture, des arts, de la littérature et de la science. Au département des manuscrits, on conserve par exemple le plus ancien texte russe daté: "Les Évangiles d'Ostromins (1056-1057), la plus importante chronique dite $\&$ Le Récit des temps reculés» (début du XIle siècle) et les manuscrits des grands hommes de la Russie. La Bibliothèque nationale est par conséquent un des plus importants dépôts des monuments littéraires russes, manuscrits et imprimés. Ses collections de livres, de périodiques et de journaux écrits dans toutes les langues parlées par les peuples de la Russie, outre le russe, lui confèrent un caractère particulièrement complet, voire unique. La Bibliothèque devient ainsi un centre des relations multinationales. 


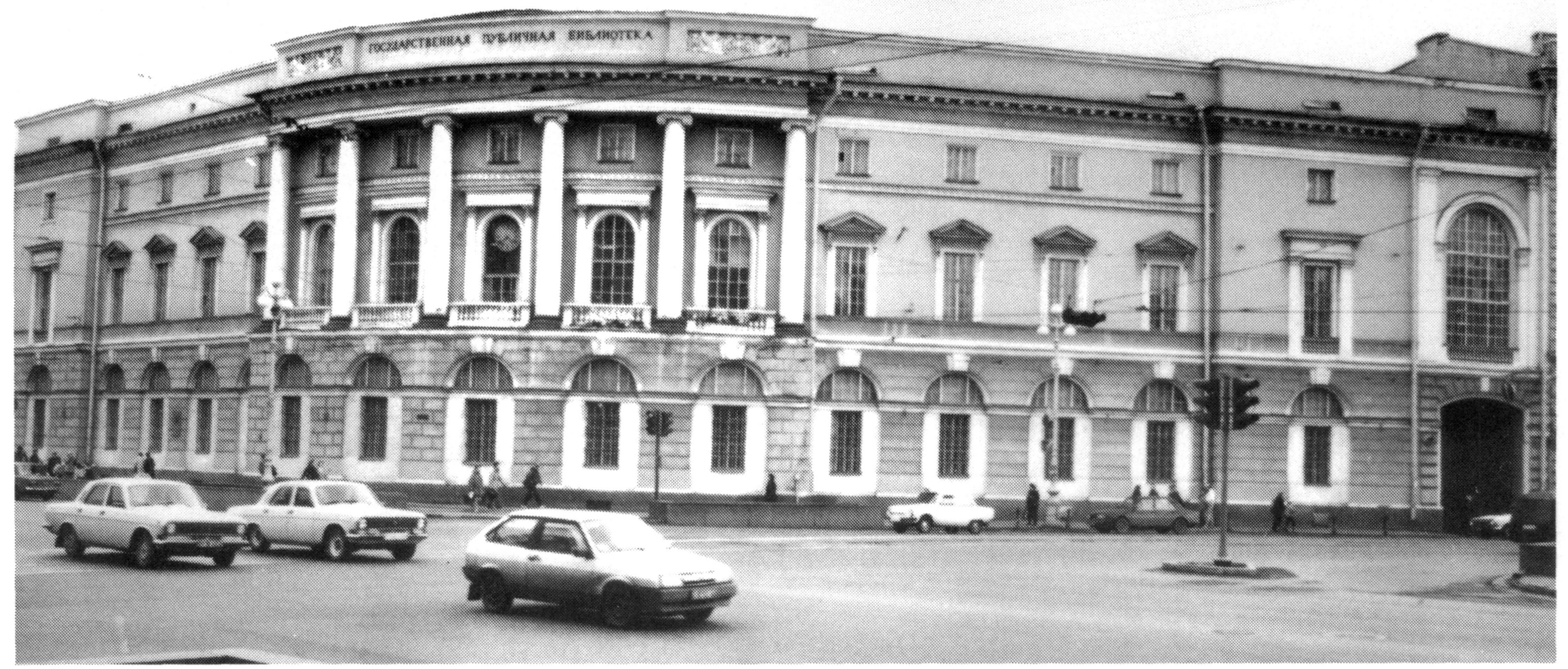

Premier édifice de la Bibliothèque nationale construit entre 1795 et 1801 à Saint-Pétersbourg

La Bibliothèque possède le fonds dit «Rossica», constitué par une collection complète d'ouvrages sur la Russie publiés dans la plupart des langues de l'Europe occidentale, depuis les témoignages des premiers voyageurs étrangers, ayant visité la Russie aux XVle et $X V I l e$ siècles, jusqu'aux écrits publiés au début du XXe siècle. Le «Rossica» n'a son semblable ni en Russie ni ailleurs, autant par la quantité que par la richesse de son répertoire. Les collections de livres et de manuscrits étrangers, tant occidentaux qu'orientaux, sont aussi très riches et très variés. Elles symbolisent les liens solides qui unissent la culture russe à celles des peuples de l'Orient, de l'Europe occidentale et de l'Amérique. II faut mentionner en particulier les documents provenant des archives de la Bastille, la bibliothèque personnelle de Voltaire, les collections des manuscrits hébreux rassemblés par FirKovitch ${ }^{1}$, la collection comportant près de 6000 incunables, les pamphlets et brochures de l'époque de la révolution anglaise du XVIlesiècle, de la Révolution française, de la Commune de Paris, etc.

L'histoire de la Bibliothèque nationale de Russie est étroitement liée à toutes les périodes historiques de notre pays. Elle témoigne de sa présence dans la société et rend compte des bouleversements provoqués par les réorganisations de l'État. Les missions de la Bibliothèque ont subi des changements en fonction des modifications territoriales du pays, survenues au long de l'histoire. Malgré tout, la Bibliothèque a conservé son statut de bibliothèque nationale.

À l'époque de la fondation de la $\mathrm{Bi}$ bliothèque, la Russie était un vaste empire qui comprenait plusieurs territoires. Ceux-ci sont devenus des républiques fédérées, y compris la Fédération de Russie, avec la formation de l'Union soviétique. Après la révolution de 1917, la Bibliothèque publique impériale est devenue Bibliothèque publique d'État (BPE), mais elle a conservé les fonctions de la bibliothèque nationale de la Fédération de Russie. En 1932, on lui a donné le nom de l'écrivain russe M.E. SaltykovChtchédrine.

En 1925, à Moscou, le gouvernement a créé, à partir de l'ancienne bibliothèque du musée Roumiantsev, la Bibliothèque d'État del'URSS V.I.Lénine (BEL), qui est devenue la Bibliothèque nationale de I'Union soviétique.

Lors des changements politiques profonds qui ont provoqué la désintégration de I'URSS et la séparation de la Fédération de Russie en tant qu'État indépendant, l'ensemble des bibliothèques en Russie a subi une restructuration importante. La Bibliothèque d'État V.I.Lénine (BEL) est devenue la Bibliothèque d'État de Russie (BER) et elle a été dotée du statut de «Bibliothèque nationale de Russie». En même temps, la Bibliothèque publique d'État M.E.Saltykov-Chtchédrine à Saint-Pétersbourg, par le décret du Président de Russie, compte tenu de ses fonctions, a été transformée en Bibliothèque nationale de Russie (BNR).

1. FirKovitch, Abraham Samonilovitch (17861874), né en Crimée, auteur de divers écrits polémiques, archéologue, hébraissant. Au cours de sa longue vie, il effectua nombre de voyages au Proche-Orient, en Asie centrale et en Égypte, en rassemblant une importante collection de manuscrits hébreux et caraītes qui n'a sa pareille nulle part. Il la légua à la Bibliothèque publique impériale (BNR) où elle est toujours conservée. 
Les processus historiques dans le pays ont donc conduit à l'existence de deux bibliothèques nationales dans la Fédération de Russie (BER et BNR). La question qui se pose concerne le mode de répartition de leurs fonctions et la coordination de leurs activités. L'expérience a démontré qu'il n'était pas nécessaire de changer radicalement leur caractère et les méthodes de travail adoptées par chacune d'elles. En activité dans le même pays (URSS) jusqu'à récemment, elles possèdent certains traits propres dans l'organisation du travail qui a pris forme au fur et à mesure du développement des bibliothèques et de la bibliothéconomie en Russie.

II faut remarquer cependant que la Bibliothèque nationale de Russie (BNR), fondée presque soixante-dix ans avant la Bibliothèque d'État de Russie, avait déjà établi certaines traditions et avait toujours acquis d'une façon exhaustive les publications nationales parues avant 1925. La Bibliothèque prête aussi une grande attention aux problèmes de la coordination des recherches en bibliothéconomie, en bibliographie et en histoire du livre en Russie, et elle offre une aide et des consultations méthodiques aux grandes bibliothèques de diverses régions du pays.

La Bibliothèque d'État de Russie (BER) présente aussi des traits particuliers. Après qu'on lui ait conféré le statut de Bibliothèque nationale d'URSS, elle a défini des priorités pour les acquisitions de publications courantes, et, ce qui est très important, pour les acquisitions de livres et périodiques étrangers. Elle reçoit, à titre de dépôt légal, une copie dactylographiée de toutes les thèses de doctorat; elle a été chargée de la coordination des activités de grandes bibliothèques des républiques fédérées, et, enfin, elle offre le meilleur potentiel d'action dans le domaine de la coopération internationale. Il est évident que, compte tenu de l'expérience de ces deux bibliothèques, de leurs possibilités immenses en tant que centres d'information, de leurs collections très riches, de leur personnel hautement qualifié, une collaboration d'égal à égal est souhaitée.

La Bibliothèque nationale de Russie éprouve actuellement de grandes difficultés à cause du manque d'espace pour les collections, de l'organisation inadéquate du service à la clientèle et d'une infrastructure technique déficiente. Ces problèmes sont aggravés par une crise économique profonde dont les effets risquent de compromettre l'existence detoutes les bibliothèques du pays.

La Bibliothèque nationale de Russie affronte toutes ces difficultés, comme les autres bibliothèques, mais sa situation est plus critique à cause de la nature particulière de sa mission. Les problèmes actuels touchent l'acquisition et son caractère d'exhaustivité propre à une bibliothèque nationale. Le mode d'acquisition principal de la Bibliothèque a toujours été, depuis 1810 , le dépôt légal avec ses deux exemplaires gratuits de toutes les publications paraissant sur le territoire de la Russie. Cette source représente environ $95 \%$ de la production nationale et $60 \%$ environ du volume des acquisitions faites par la Bibliothèque nationale. Les $40 \%$ qui restent sont constitués d'exemplaires supplémentaires achetés pour les fonds auxiliaires et pour les échanges internationaux de publications. Ces dernières années, la publication d'ouvrages a nettement diminué par suite d'une augmentation continue du prix du papier et des services d'impression. Cette diminution de la production des publications se répercute sur les acquisitions de la Bibliothèque nationale: elle a reçu presque deux fois moins d'ouvrages en 1992 qu'en 1986 , soit 376000 unités physiques. En 1991, elle avait reçu 550000 unités physiques.

Parallèlement à ce processus, on observe des changements profonds dans les catégories de publications. Ainsi, il faut noter l'accroissement de parutions qu'on peut qualifier de «littérature commerciale». Viennent ensuite les publications en philosophie, en religion et en sciences occultes. On observe aussi une diminution de la production d'ouvrages de référence, d'ouvrages en sciences naturelles, en techniques, en littérature classique et en poésie. Les tirages des périodiques ont aussi chuté sensiblement. On assiste donc à la dégradation progressive du système qui réglait auparavant la répartition du dépót légal. Les contrôles législatifs sont insuffisants et mal adaptés aux changements. Le dépôt légal effectué par les républiques de l'ancienne URSS n'existe pratiquement plus.
Il en va de même pour les acquisitions de publications étrangères. La Bibliothèque nationale de Russie a toujours acquis des ouvrages publiés dans toutes les langues de l'Europe, de l'Asie et de l'Afrique. Ces acquisitions couvraient diverses catégories, comme les ouvrages de référence, la médecine, les sciences et les techniques, les sciences sociales, les humanités (belles-lettres, linguistique), les beaux-arts. Pendant plusieurs décennies, les acquisitions et les échanges internationaux ont été des sources essentielles. Mais depuis deux ans, la Bibliothèque nationale de Russie est privée de la possibilité d'acquérir des publications étrangères à cause de l'absence totale de crédits. L'estimation sommaire des besoins annuels pour l'acquisition des livres les plus importants et l'abonnement aux périodiques s'élève à au moins un million de dollars américains. Les échanges internationaux de publications demeurent donc actuellement le seul moyen pratique d'acquérir des ouvrages étrangers. Par ces échanges, la Bibliothèque reçoit annuellement 7000 à 8000 livres, ainsi qu'environ 30000 numéros de périodiques pour compléter le fonds étranger. II s'agit surtout des publications des pays industrialisés. C'est pourquoi la Bibliothèque s'intéresse beaucoup aux échanges de publications (livres et périodiques) avec les bibliothèques et les organismes à l'étranger. Al'heure actuelle, elle compte plus de 2500 partenaires dans 110 pays. Les bibliothèques étrangères acceptent volontiers les échanges en matière d'histoire, de linguistique et de belles-lettres, mais il est très difficile d'obtenir les publications en sciences et en techniques (livres et périodiques), ainsi que les ouvrages de référence en sciences exactes et en sciences naturelles. Le problème majeur de la Bibliothèque nationale de Russie demeure donc lié aux acquisitions et au développement des collections

Au cours d'une année, la Bibliothèque sert plus de 1300000 lecteurs en leur communiquant quelque 11000000 d'unités physiques. La majorité de la clientèle est constituée de chercheurs et de spécialistes divers, mais on admet aussi des étudiants, des retraités, des élèves des grandes classes. L'an dernier, des lecteurs venus de 88 pays ont visité la Bibliothèque. La communication des ouvrages 
dans les salles de lecture, qui comptent 1390 places, est le mode habituel du service à la clientèle. Le lecteur peut souscrire à l'abonnement payant pour demander les livres par téléphone. On pratique à la Bibliothèque un service adapté qui tient compte des caractéristiques d'áge et des niveaux de formation des lecteurs. C'est ainsi qu'on dispose de salles pour les chercheurs, de salles spécialisées et de salles pour les lecteurs ordinaires, enfin, de salles pour les adolescents. D'autres services sont offerts tels que l'abonnement individuel avec prêt à domicile dont peuvent bénéficier les chercheurs ayant le grade de docteur d'État (quelque 60000 communications par an), le microfilmage sur demande (2 000000 d'images par an), le microfichage (200 000 unités par an), les photocopies et les photos (plus d'un million de copies), les traductions des textes étrangers (17 000 pages par an).

Compte tenu des demandes de bibliothèques, aussi bien celles de l'intérieur du pays que celles de l'étranger, la Bibliothèque nationale de Russie effectue le prêt entre bibliothèques. Malheureusement, ces derniers temps, elle n'était plus en mesure de satisfaire un grand nombre de demandes à cause de l'absence des ouvrages dans ses collections. Ce problème touche surtout les ouvrages étrangers, livres ou périodiques, que la Bibliothèque n'a pas réussi à acquérir ou à recevoir dans le cadre des échanges internationaux. Le volume du prêt entre bibliothèques, y compris le prêt international, a été sensiblement réduit à cause de l'augmentation progressive des frais postaux et parce que plusieurs pays exigent des coupons qu'il faut payer en devises fortes pour avoir le droit de recevoir les ouvrages en contrepartie, en guise de prêt international. Bien sûr, tous ces aspects compromettent les services aux lecteurs.

Afin de remédier à cette situation et d'atteindre un niveau de service plus satisfaisant, la Bibliothèque nationale de Russie tâche d'apporter des changements dans l'utilisation des technologies traditionnelles, en procédant à l'informatisation et au renouvellement de son équipement. Mais il ne s'agit là que de la première étape d'un long itinéraire. Le personnel travaille au projet d'un système informatique intégré de la Bibliothèque (SIIB) qui prévoit la création graduelle de sous-systèmes du SIIB et le branchement ultérieur au système global de la Russie, suivi de l'accès au système mondial d'information. Pour réaliser ce travail, il faut créer au moins 800 postes informatisés.

À l'heure actuelle, la Bibliothèque nationale de Russie dispose d'une soixantaine de postes informatisés. En même temps, les essais d'exploitation de certains sous-systèmes informatiques sont effectués en visant la réalisation des objectifs locaux. Les travaux de création des catalogues électroniques sont amorcés. Ainsi, on a introduit dans la base de données relative au fonds de documentation technique et de brevets quelque 90000 enregistrements; la base de données concernant Léningrad-SaintPétersbourg en a plus de 30000 . La réalisation de ce genre de travaux est malheureusement freinée par un faible financement de ces programmes, par un manque évident de matériel, de logiciels et des spécialistes qualifiés.

De grandes difficultés ont surgi lors de la création d'un réseau interbibliothèques d'informations couvrant toute la Russie, réseau auquel pourraient être branchées également des bibliothèques étrangères. La Bibliothèque nationale de Russie effectue des recherches sur la coordination des formats d'enregistrements, des vedettes-matière, des moyens techniques et des logiciels compatibles. Malheureusement, jusqu'à présent, la Russie ne dispose pas de programme général d'informatisation qui réunisse toutes les bibliothèques et, par conséquent, plusieurs d'entre elles créent leurs programmes spécifiques sans souci de compatibilité.

La Bibliothèque nationale de Russie cherche à tenir compte de l'expérience de ses collègues à l'étranger. À cette fin, elle élargit ses contacts et favorise la coopération avec d'autres bibliothèques et organismes du monde entier, tant sur la base des conventions bilatérales que dans le cadre d'activités des organisations internationales. Peu auparavant, la Bibliothèque nationale de Russie entretenait des liens étroits fondés sur des accords à long terme avec les bibliothèques de l'Europe centrale (République démocratique allemande, Bulgarie, Hongrie, Tchécoslovaquie, Pologne, Yougoslavie) tout en participant à certaines manifestations des organisations internationales. Actuellement, les contacts avec les bibliothèques des anciens pays socialistes se sont considérablement réduits, alors que les contacts avec d'autres bibliothèques ne sont pas encore suffisamment développés. Cependant, on a su garder des liens étroits avec quelques anciens partenaires, bibliotheques nationales telles que celles de la Pologne, de la République tchèque, de la Tchernogorie. Les échanges avecla Finlande se poursuivent avec succès. II faut noter les nouvelles conventions signées avec la Bibliothèque nationale du Québec et la Bibliothèque nationale d'Israël. En 1993, la Bibliothèque nationale de Russie doit prendre part à une grande exposition qui sera réalisée sous les auspices de la Bibliothèque nationale de Paris. En 1990 et 1991, la Bibliothèque nationale de Russie a participé aux sessions annuelles de travail de l'ABSDOS. En 1992, elle participa aux travaux des congrès de l'IFLA et de LIBER. Elle espère élargir encore ses contacts avec les bibliothèques du monde entier.

Actuellement, la Bibliothèque nationale de Russie est entrée dans une nouvelle phase de son développement, caractérisée non seulement par les changements profonds que notre pays subit, mais aussi par ceux qui déterminent aujourd'hui les activités bibliothéconomiques dans le monde. Ainsi, un projet préliminaire a été élaboré pour le développement de la Bibliothèque jusqu'à l'an 2005. Ce projet prend en considération la réalisation plus suivie des fonctions sociales de l'organisme, son rééquipement et la mise en oeuvre des technologies appliquées aux systèmes bibliothéconomiques et informatiques, un renforcement de sa base matérielle par la construction des nouveaux édifices et la reconstruction de ceux qui existent déjà, par le développement de l'infrastructure sociale, enfin par la garantie législative de ses activités en tant que bibliothèque nationale.

Les bibliothèques nationales démontrent par leur histoire qu'elles ne peuvent interrompre leur développement, leurs collections étant la chronique 


\section{Documentation et bibliothèques}

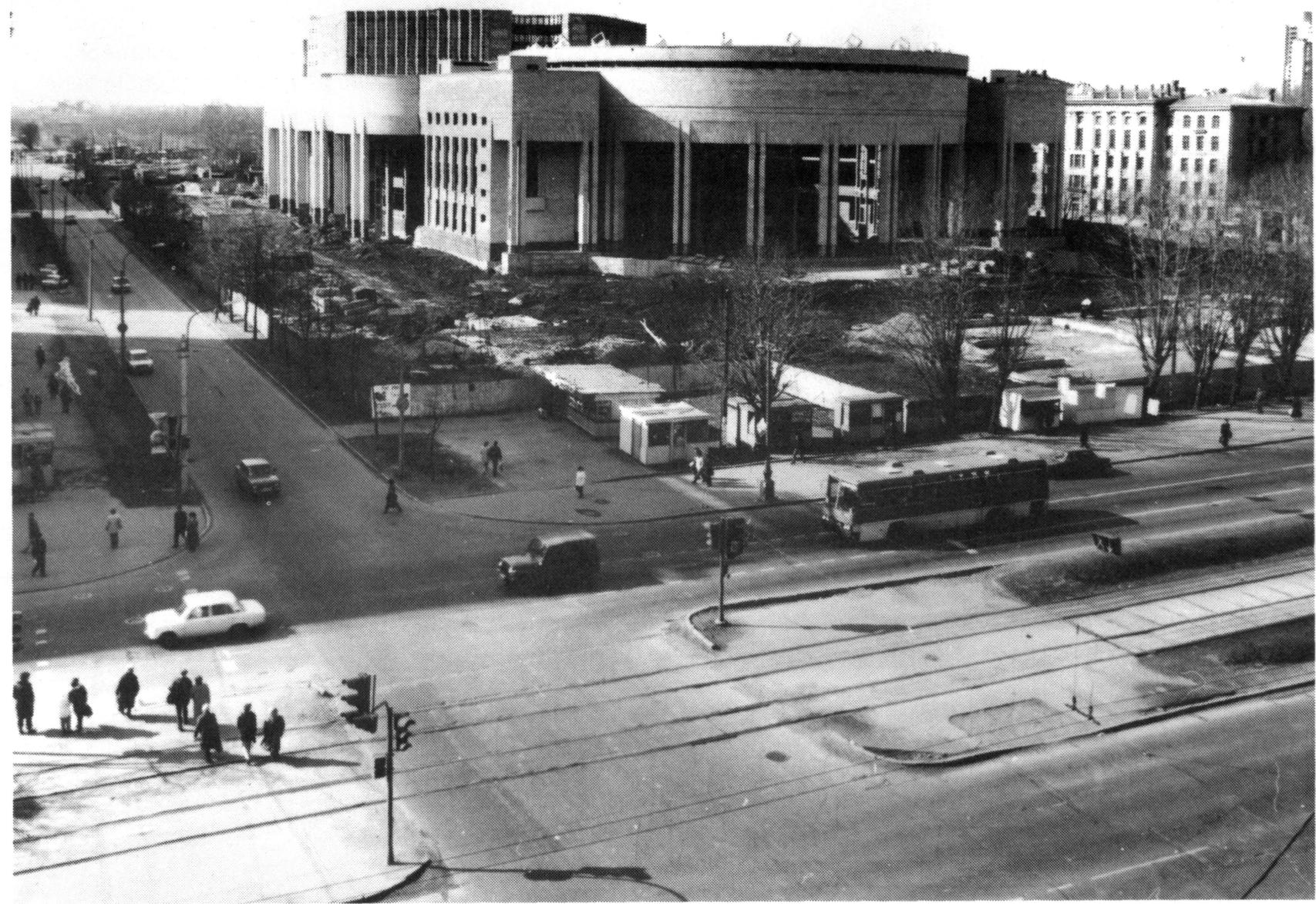

Etat des travaux de construction du nouvel édifice de la Bibliothèque nationale à Saint-Pétersbourg.

documentaire de la vie intellectuelle de la société à laquelle elles appartiennent. Les collections accusent donc une croissance constante et la vocation des bibliothèques nationales réside dans la conservation de ce potentiel intellectuel. L'accomplissement de ce mandat est déterminé dans une grande mesure par la qualité de l'infrastructure et par la technique nécessaires.

Au début des années soixante-dix, la Bibliothèquenationalea connu une situation extrêmement difficile: il a fallu entreprendre la réparation majeure d'un des grands bâtiments de la bibliothèque, ce qui a provoqué sur le champ une pénurie de locaux pour le stockage des ouvrages. Les espaces que la ville a accordés à ces fins étaient insuffisants et mal aménagés pour la mise en service. La situation était telle que les collections de la bibliothèque ont été dispersées dans quatorze endroits différents situés dans les neuf arrondissements de Léningrad. Devant ces difficultés, le gouvernement a décrété la construction d'un nouvel édifice. Les travaux ont commencé en 1985 . Le projet adopté prévoit un bâtiment conçu spécialement pour loger la Bibliothèque nationale et doté d'un ensemble de locaux. $\mathrm{Ce}$ bâtiment abritera les dépôts qui pourront recevoir 20 millions d'ouvrages et les salles de lecture qui pourront accueillir
2000 lecteurs. Les travaux sont répartis en deux étapes: la première aboutira à l'aménagement des dépôts pour la conservation de dix millions d'unités physiques et de toutes les salles de lecture; la deuxième étape sera complétée par l'achèvement de la construction de l'autre moitié des dépôts de la même capacité que le premier, et par la mise en fonction des locaux des services techniques. Malheureusement, les travaux accusent un grand retard par rapport au programme de construction. II était prévu que la première étape fût terminée en 1990 . La nouvelle échéance est fixée à 1994, et l'ouverture devra avoir lieu en 1995 . En 1992, les travaux relatifs à la deuxième 
étape ont commencé. Lorsque le nouvel édifice aura été construit, la Bibliothèque nationale sera en mesure de mieux accomplir l'ensemble de ses fonctions propres.

En même temps, la Bibliothèque nationale ressent avec évidence la nécessité d'une législation nouvelle qui puisse la régir et la protéger. Une telle bibliothèque ne peut se développer avec succès sans un soutien constant de la part de l'État. La Bibliothèque a déjà fait adopter par le gouvernement de I'URSS et, plus tard, par celui de la Russie, certains actes prévoyant des mesures pour un renforcement de son infrastructure matérielle et de ses moyens techniques. Récemment, dans le but de préserver les plus grands centres culturels du patrimoine, le nouveau gouvernement de la Russie a juridiquement reconnu la catégorie des *centres culturels les plus importants du patrimoine des peuples de la Fédération de la Russien, parmi lesquels il a inclus la Bibliothèque nationale de Russie. Conformément au décret du gouvernement, la Bibliotheque nationale peut jouir d'un certain nombre de privilèges quant à son financement et quant aux salaires de son personnel; elle profite d'une indépendance élargie dans sa gestion et son directeur général est nommé par le gouvernement. II s'agit d'une garantie de l'État pour le développement et la sauvegarde des principales institutions culturelles, surtout en pleine crise économique. Afin de donner un nouvel essor à la cause des bibliothèques en Russie, les spécialistes de la Bibliothèque nationale, en collaboration avec leurs collègues, participent à l'élaboration de toute une série d'actes législatifs. Actuellement, on a remis au Soviet suprême de Russie, pour discussion, les projets de loi «Sur les bibliothèques", "Sur les bibliothèques nationales de toute la Russie» et $\kappa$ Sur les exemplaires gratuits du dépôt légal». Leur adoption sera sans doute bénéfique pour les bibliothèques du pays.

En guise de conclusion on peut affirmer que la plus ancienne bibliothèque de la Russie, à la veille de son bicentenaire, vit sa deuxième jeunesse et regarde l'avenir avec confiance.

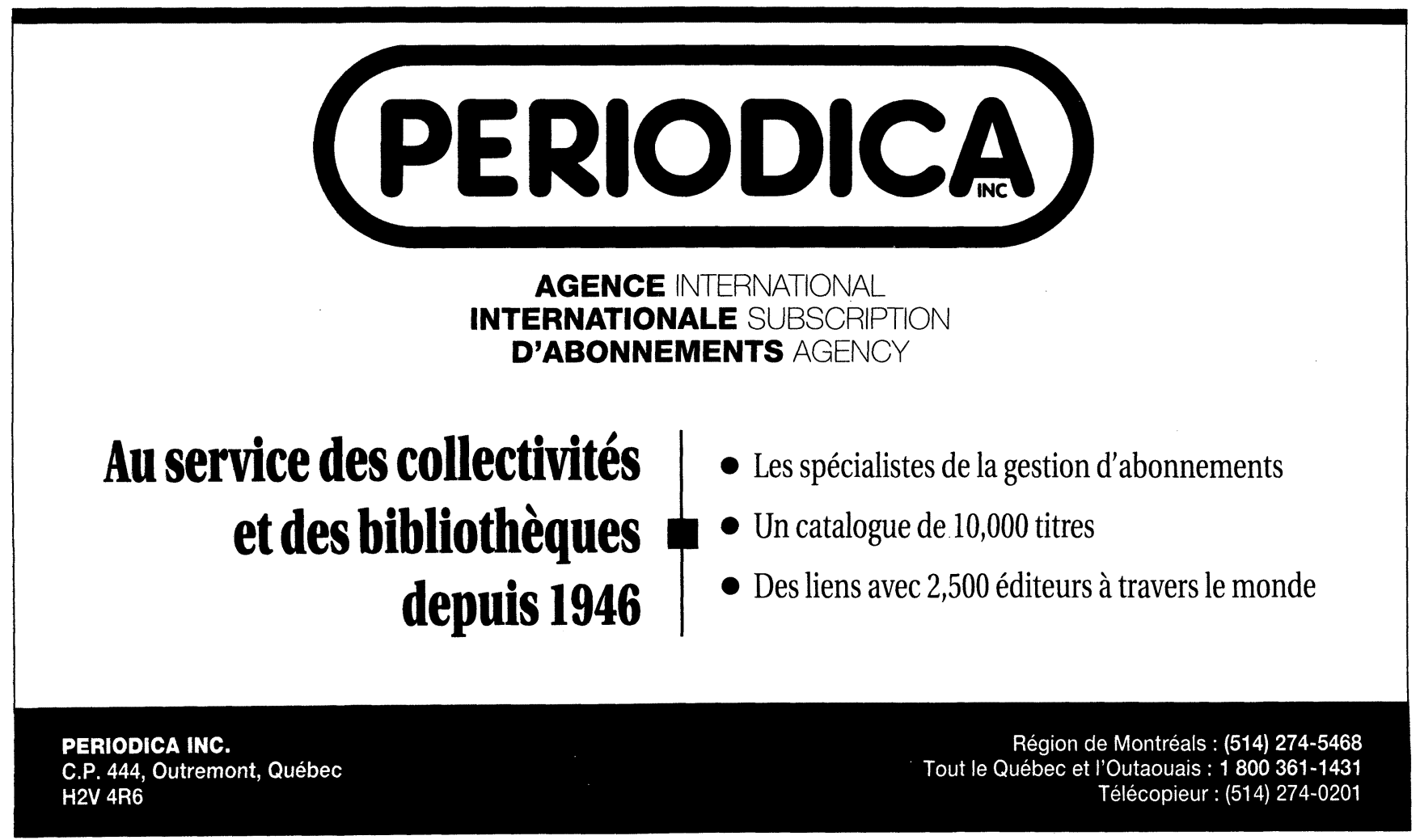

